\title{
Design condition of a sustainable two-layer circular tube with energy absorbing capacity
}

\author{
A. Nomura ${ }^{1}$, E. Ueda ${ }^{2}$, T. Wada ${ }^{1}$ \& S. Enoki ${ }^{1}$ \\ ${ }^{1}$ Department of Mechanical Engineering, \\ Nara National College of Technology, Japan \\ ${ }^{2}$ Department of Control Engineering, \\ Nara National College of Technology, Japan
}

\begin{abstract}
Recently, there have been a lot of earthquakes in Japan. Therefore, it is necessary to attach seismic isolation devices to detached houses. However, almost all detached houses do not have any seismic isolation devices attached, because the seismic isolation devices are expensive. Therefore, it is necessary to develop a low-cost seismic isolation device. We created a new energy absorbing device filled with low rigidity material in two-layer circular tubes. If hysteresis is produced in the two-layer circular tube under lateral compression load, we think that a two-layer circular tube can have energy absorbing capacity. Therefore, we think that the device has a high energy absorbing capacity. For hysteresis to occur, friction should exist between the outer layer and inner layer. Also, we should reveal a force of elastic limit to have sustainability. In this paper, in the case that outer layer is made of stainless steel and inner layer is made of acrylic plastic, we evaluated the deformation and the stress of each circular tube. As a result, we were able to clarify the relationship between the contact condition that friction occurs in each circular tube and external force of elastic limit.

Keywords: seismic isolation device, two-layer circular tube, contact condition, sustainability, energy absorbing capacity.
\end{abstract}

\section{Introduction}

Recently, there have been a lot of earthquakes in Japan. Therefore, we should develop immediately a seismic technology for saving our lives from the earthquakes. It has already developed a seismic isolation device using seismic 
isolation rubber for tower buildings and others [1, 2]. However, almost all detached houses do not have seismic isolation devices attached, because the seismic isolation devices are expensive. Therefore, it is necessary to develop a low-cost seismic isolation device.

We conducted research in order to create and test a composite material for the low-cost seismic isolation device. The composite material is composed of metal square lattice filled with low rigidity material [3]. As a result, it was able to clarify that the composite material has energy absorbing capacity. However, the composite material had little energy absorbing capacity when sustainability was considered [4].

In this research, we changed to two-layer circular tubes from metal square lattice to improve sustainability. Therefore, we created a new energy absorbing device filled with low rigidity material in two-layer circular tubes. If hysteresis is produced in the two-layer circular tube under lateral compression load, we think that two-layer circular tube can have energy absorbing capacity. Therefore, we think that the device has high energy absorbing capacity. For hysteresis to occur, friction should exist between outer layer and inner layer. Also, we should reveal a force of elastic limit to have sustainability. In this paper, in the case that outer layer is made of stainless steel and inner layer is made of acrylic plastic, we evaluated the deformation and the stress of each circular tube.

\section{Two-layer circular tube}

We create a new seismic isolation device that does not depend on load directions for high energy absorbing capacity. A two-layer circular tube is a unit of the device. An example of the device is shown in fig. 1(a). In this case, all of the

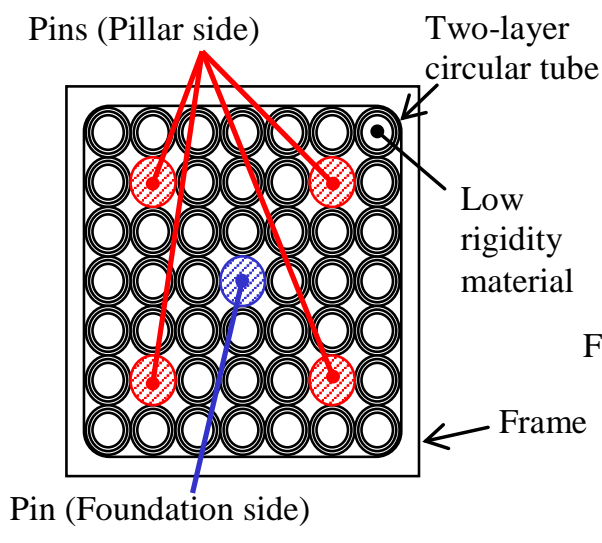

(a) An example of a created seismic isolation device.

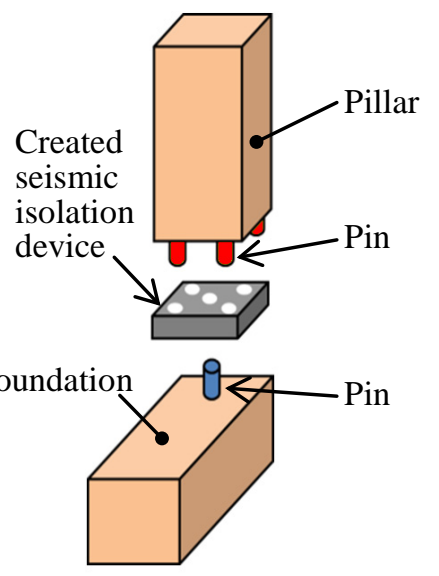

(b) Application of a created seismic isolation device.

Figure 1: Created seismic isolation device by using two-layer circular tubes. 
two-layer circular tubes are the same in size. The device is put between a foundation and a pillar by using connecting pins in a detached house (as shown in fig. 1(b)).

Seismic energy is transmitted from the foundation to the pillar through the connecting pins and the two-layer circular tubes. The two-layer circular tube is subjected to lateral compression load. We can design numbers of the circular tubes and the size of each circular tube for necessary energy absorbing capacity if we know properties of the two-layer circular tube under lateral compression load.

Therefore we research properties of the two-layer circular tube under lateral compression load. The two-layer circular tube is shown in fig. 2(a). In this paper, the outer layer is made of stainless steel and the inner layer is made of acrylic plastic. The two-layer circular tube under lateral compression load is shown in fig. 2(b). The device is sustainable in the case that the all circular tubes deform in elastic region. Friction exists between the outer layer and the inner layer and the two-layer circular tube has high energy absorbing capacity, in the case that the inner layer contacts with the outer layer.

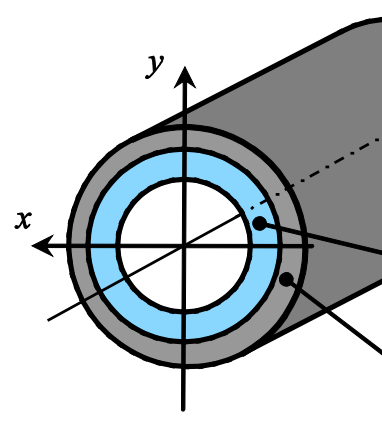

(a) Two-layer circular tube

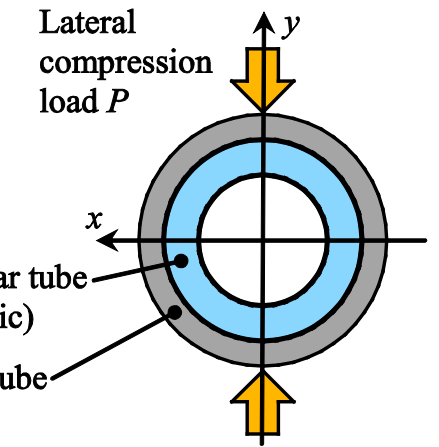

(b) Two-layer circular tube in device

Figure 2: $\quad$ A unit of created seismic isolation device.

\section{Contact condition of a two-layer circular tube by using a single circular tube model}

A single circular tube model is used for considering a contact condition between the outer layer and the inner layer under lateral compression load. We focus deformation amount in vertical direction to the lateral compression load direction. We think that the inner layer contacts with the outer layer in the case that the displacement of the inner layer is bigger than the displacement of the outer layer. 


\subsection{Single circular tube model}

In this section, we derive deformation amount in vertical direction to lateral compression load direction, deformation amount in lateral compression load direction and the maximum bending stress $\sigma_{\max }$ by using the single circular tube model. A section of a single circular tube is shown in fig. 3(a), the 1/4 model is shown in fig. 3(b). Internal bending moment $M$ acts on an imaginary crosssection which is cut at any angle $\theta$ in fig. 3(b). A free body of single circular tube is shown in fig. 3(c). $M_{0}$ is the internal moment at an angle $\theta$ of $0^{\circ}$ and $P^{\prime}$ is an imaginary load. The internal moment $M$ is derived as eqn (1) using moment equilibrium on the imaginary cross-section.

$$
M=M_{o}+\frac{P}{2}(r-r \cos \theta)+\frac{P^{\prime}}{2} r \sin \theta
$$

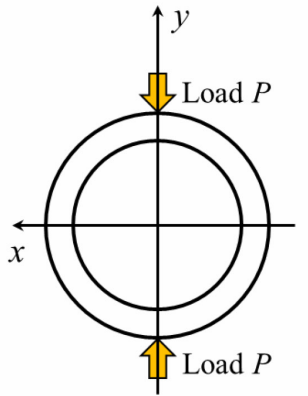

(a)

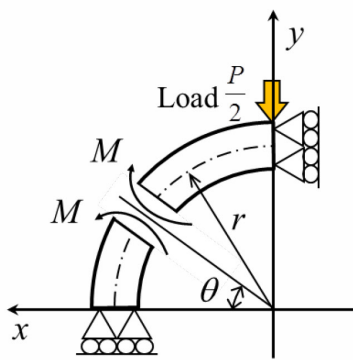

(b)

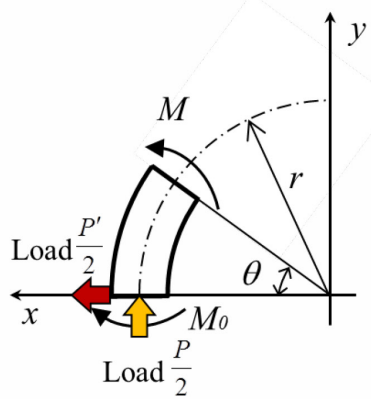

(c)

Figure 3: A single circular tube model for a two-layer circular tube under lateral compression load. (a) A section of a single circular tube; (b) 1/4 model of a single circular tube; (c) A free body of a single circular tube.

Slope deflection $\phi$ at an angle $\theta$ of $0^{\circ}$ is derived as eqn (2) using Castigliano's theorem, where $U$ is the strain energy, $E$ is the Young's modulus and $I$ is the second moment of area.

$$
\phi=\frac{\partial U}{\partial M_{o}}=\frac{\partial U}{\partial M} \frac{\partial M}{\partial M_{o}}=\frac{\partial}{\partial M}\left(\int_{0}^{\frac{\pi}{2}} \frac{M^{2}}{2 E I} r d \theta\right) \cdot \frac{\partial M}{\partial M_{o}}
$$

The slope deflection $\phi$ is zero. The internal moment $M_{0}$ is derived as eqn (3) by using eqn (2).

$$
M_{o}=-\frac{\operatorname{Pr}}{\pi}\left(\frac{\pi}{2}-1\right)-\frac{P^{\prime} r}{\pi}
$$

The imaginary load $P^{\prime}$ should be zero. The internal bending moment $M$ is derived as eqn (4) by using eqn (1) and eqn (3). 


$$
M=-\frac{\operatorname{Pr}}{2}\left(\cos \theta-\frac{2}{\pi}\right)
$$

At first, we derive the maximum bending stress $\sigma_{\max }$. Fig. 4 shows a relationship between the angle $\theta$ and the internal bending moment $M$ in the case that the compression load $P$ and the radius $r$ of tube wall thickness centreline are constant. It is clarified that the internal bending moment $M$ becomes maximum at an angle $\theta$ of $90^{\circ}$ as shown in Fig. 4. The maximum internal bending moment $M_{\max }$ is given by eqn (5).

$$
M_{\max }=\frac{P \cdot r}{\pi}
$$

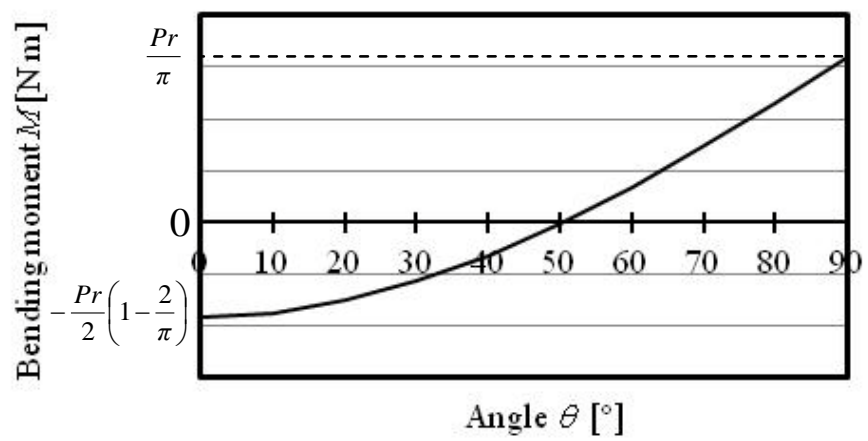

Figure 4: $\quad$ Relationship between angle $\theta$ and internal bending moment $M$.

The maximum bending stress $\sigma_{\max }$ is derived as eqn (6) by using eqn (5),

$$
\sigma_{\max }=\frac{M_{\max }}{I} \cdot \frac{t}{2}=\frac{P \cdot r \cdot t}{2 \pi \cdot I}
$$

where $t$ is tube wall thickness and $I$ is second moment of area.

Secondly, $y$ axial deformation amount $\delta_{y}$ is derived as eqn (7) and $x$ axial deformation amount $\delta_{x}$ is derived as eqn (8) by using Castigliano's theorem in fig. 3 (b).

$$
\begin{aligned}
& \delta_{y}=\frac{P \cdot r^{3}}{E \cdot I}\left(\frac{\pi}{4}-\frac{2}{\pi}\right) \\
& \delta_{x}=\frac{P \cdot r^{3}}{E \cdot I}\left(\frac{2}{\pi}-\frac{1}{2}\right)
\end{aligned}
$$

\subsection{Contact condition between outer layer and inner layer}

In this section, we consider contact condition between the outer layer and the inner layer in two-layer circular tube using the $x$ axial deformation amount $\delta_{x}$ evaluated using the single circular tube model. Deformations of the two-layer circular tube are shown in fig. 5. The $y$ axial deformation amount $\delta_{y 1}$ of the outer layer is same the $y$ axial deformation amount $\delta_{y 2}$ of the inner layer. And the load share $P_{1}$ of the outer layer and the load share $P_{2}$ of the inner layer are different as shown in fig. 6. 

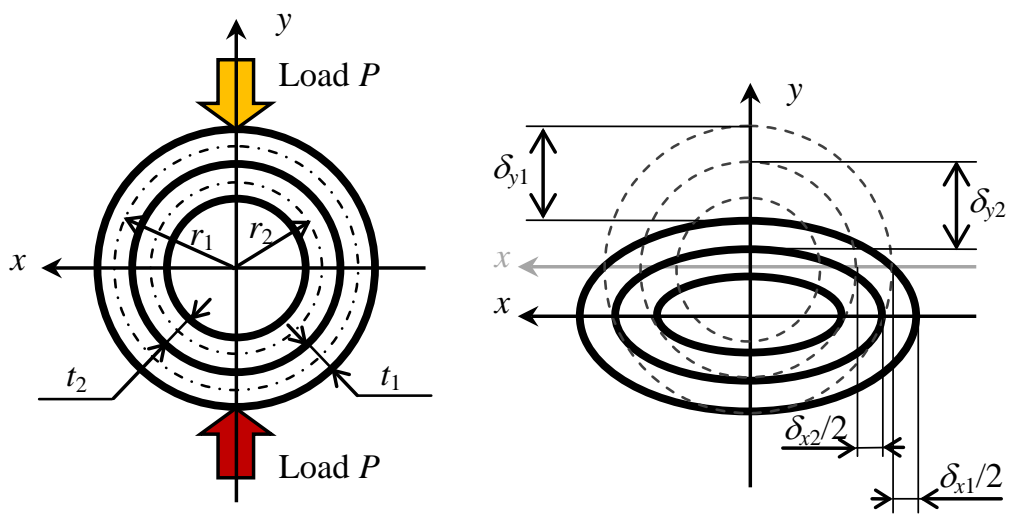

Figure 5: $\quad$ Deformation of a two-layer circular tube under lateral compression load.

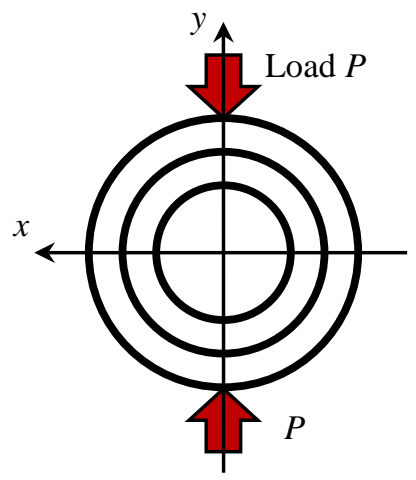

(a) Two-layer circular tube

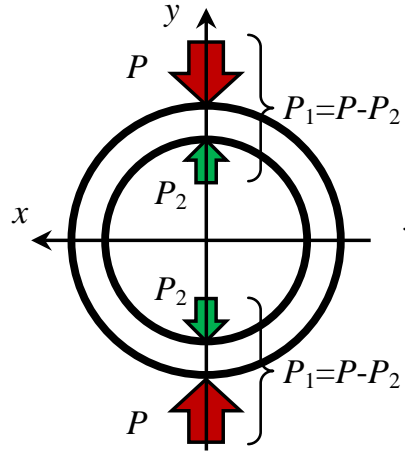

(b) Outer layer

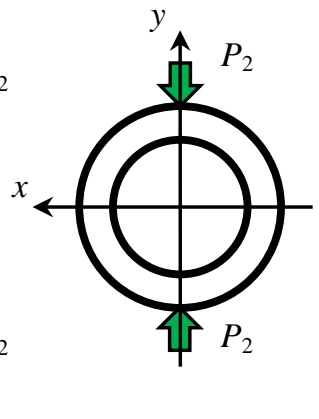

(c) Inner layer

Figure 6: $\quad$ Load share in a two-layer circular tube.

By using eqn (7), the $y$ axial deformation amounts $\delta_{y 1}$ of the outer layer and $\delta_{y 2}$ of the inner layer are given by

$$
\delta_{y 1}=\frac{P_{1} \cdot r_{1}^{3}}{E_{1} \cdot I_{1}}\left(\frac{\pi}{4}-\frac{2}{\pi}\right) \quad \delta_{y 2}=\frac{P_{2} \cdot r_{2}^{3}}{E_{2} \cdot I_{2}}\left(\frac{\pi}{4}-\frac{2}{\pi}\right)
$$

where $E_{1}$ is young's modulus of the outer layer, $E_{2}$ is young's modulus of the inner layer, $I_{1}$ is second moment of area of outer layer and $I_{2}$ is second moment of area of the inner layer. Considering $\delta_{y 1}=\delta_{y 2}$, the relationship between $P_{1}$ and $P_{2}$ is derived as eqn (10).

$$
P_{1}=\frac{E_{1} \cdot I_{1}}{E_{2} \cdot I_{2}} \frac{r_{2}^{3}}{r_{1}^{3}} P_{2}
$$

Similarly, we obtain the $x$ axial deformation amount $\delta_{x 1}$ of outer layer and the $x$ axial deformation amount $\delta_{x 2}$ of inner layer by using eqn (8). 


$$
\delta_{x 1}=\frac{P_{1} \cdot r_{1}^{3}}{E_{1} \cdot I_{1}}\left(\frac{2}{\pi}-\frac{1}{2}\right) \quad \delta_{x 2}=\frac{P_{2} \cdot r_{2}^{3}}{E_{2} \cdot I_{2}}\left(\frac{2}{\pi}-\frac{1}{2}\right)
$$

By substituting eqn (10) into eqn (11), we obtain the following equation.

$$
\delta_{x 1}=\delta_{x 2}
$$

Eqn (12) means that contact between the inner layer and the outer layer before loading continues after loading. We think that the inner layer contacts with outer layer all over the circumference if the inner layer contacts with the outer layer on $x$ axis and $y$ axis.

\subsection{Sustainability of two-layer circular tube}

In this section, we conduct the maximum $y$ axial deformation amount $\delta_{y \max }$ in elastic region. Design condition in elastic region is that the maximum bending stress $\sigma_{\max }$ must be smaller than yield strength $\sigma_{a}$. Therefore, the allowable lateral compression load $P_{a}$ is derived as eqn (13) by using eqn (6).

$$
P_{\mathrm{a}}=\frac{2 \pi \cdot I \cdot \sigma_{a}}{r \cdot t}
$$

Considering relationship between the allowable $y$ axial deformation amount $\delta_{y a}$ and the yield strength $\sigma_{a}$ by using eqn (13) and eqn (9), we obtain eqn (14).

$$
\delta_{y a}=\frac{2 \pi \cdot \sigma_{a} \cdot r^{2}}{E \cdot t}\left(\frac{\pi}{4}-\frac{2}{\pi}\right)
$$

where $E$ is young's modulus, $t$ is tube wall thickness and $r$ is radius of circular tube thickness centreline. Radius $r_{1}$ of outer layer thickness centreline and radius $r_{2}$ inner layer thickness centreline are given by

$$
r_{1}=\frac{1}{2}\left(d_{1}-t_{1}\right) \quad r_{2}=\frac{1}{2}\left(d_{1}-2 t_{1}-t_{2}\right)
$$

By substituting eqn (15) into eqn (14), we obtain the allowable deformation amount $\delta_{y a 1}$ of outer layer and the allowable deformation amount $\delta_{y a 2}$ of the inner layer.

$$
\begin{gathered}
\delta_{y a 1}=\frac{\pi \cdot \sigma_{a 1}\left(d_{1}-t_{1}\right)^{2}}{2 E_{1} \cdot t_{1}}\left(\frac{\pi}{4}-\frac{2}{\pi}\right) \\
\delta_{y a 2}=\frac{\pi \cdot \sigma_{a 2}\left(d_{1}-2 t_{1}-t_{2}\right)^{2}}{2 E_{2} \cdot t_{2}}\left(\frac{\pi}{4}-\frac{2}{\pi}\right)
\end{gathered}
$$

Second moment of area $I_{1}$ and $I_{2}$ of the outer layer and the inner layer are shown as

$$
I_{1}=\frac{t_{1}^{3} \cdot l}{12} \quad I_{2}=\frac{t_{2}^{3} \cdot l}{12}
$$

where $l$ is length of the two-layer circular tube. Therefore, by substituting eqn (18) into eqn (13), we obtain the allowable load $P_{a 2}$ of inner layer and the allowable load $P_{a 1}$ of outer layer.

$$
P_{a 1}=\frac{\pi \cdot \sigma_{a 1} \cdot t_{1}^{2} \cdot l}{3\left(d_{1}-t_{1}\right)}
$$




$$
P_{a 2}=\frac{\pi \cdot \sigma_{a 2} \cdot t_{2}^{2} \cdot l}{3\left(d_{1}-2 t_{1}-t_{2}\right)}
$$

Design conditions of the two-layer circular tube in consideration of sustainability are that force acting on the circular tube is smaller than the allowable load as shown eqn (19) and eqn (20) and that the $y$ axial deformation amount is smaller than the allowable deformation amount as shown eqn (16) and eqn (17).

\section{Effect of friction for contact condition in two-layer circular tube}

We conducted the contact condition of the two-layer circular tube under lateral compression load in consideration of sustainability in chapter 3. However, the single circular tube model, which was used in the consideration, is not to consider friction. Therefore, we review the contact condition in consideration of the friction by using Finite Element Analysis (FEA) in this chapter.

\subsection{Design object and analysis method}

Materials and sizes of the design object shows in table 1. FE analyses were carried out for this design object in consideration of the friction. FEA software ANSYS 12.0 (ANSYS Inc.) was used for the analyses.

Table 1: $\quad$ Materials and sizes of two-layer circular tube (design object).

\begin{tabular}{|c|c|c|}
\hline & Outer layer & Inner layer \\
\hline Material & Stainless Steel & Acrylic Plastic \\
\hline Young's modulus (MPa) & $E_{1}=1.93 \times 10^{5}$ & $E_{2}=3.14 \times 10^{3}$ \\
\hline $\begin{array}{c}\text { Poisson's ratio } \\
\text { Yield strength }(\mathrm{MPa})\end{array}$ & $v_{1}=0.28$ & $v_{2}=0.35$ \\
\hline Outer diameter & $\sigma_{a 1}=205$ & $\sigma_{a 2}=50$ \\
\hline Sizes $(\mathrm{mm})$ & $d_{1}=20$ & $\left(d_{2}=19\right)$ \\
& Tube wall thickness \\
Length & $t_{1}=0.5$ & $t_{2}=3.5$ \\
\hline
\end{tabular}

FE model of the design object shows in fig. 7. The model was 2-D symmetrical model. Element type was solid element with 8 nodes and the mean element size was $0.2 \mathrm{~mm}$. Mesh division shows in fig. 7(a). The analyses were carried out as plane stress problem. Boundary condition was as shown in fig. 7 (b). Coefficient of friction $\mu$ was set to 0.5 as contact condition. Constraint displacement was set at nodes on upper side of elastic body 1 in $y$ axial direction. 


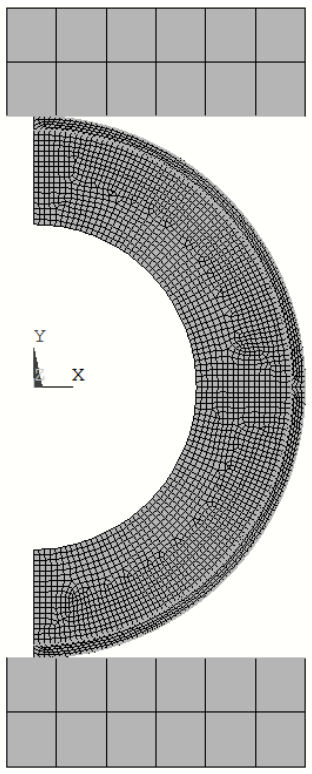

(a) Mesh division

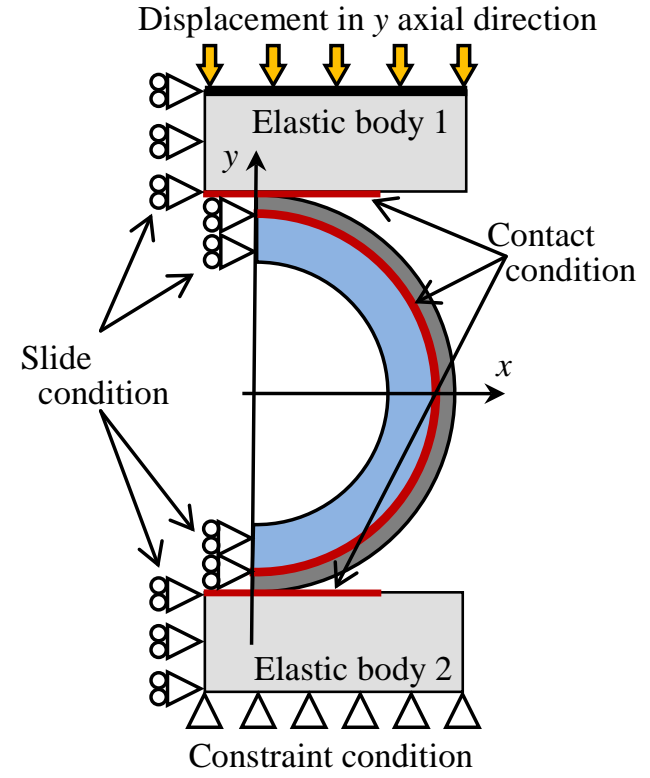

(b) Boundary conditions

Figure 7: $\quad$ FE model of two-layer circular tube. (Number of elements: 3526, Number of nodes: 9791).

\subsection{Effect of friction for contact condition}

The constraint displacements were set so that the $y$ axial deformation amount of the two-layer circular tube became $0.188 \mathrm{~mm}$ and $0.100 \mathrm{~mm}$. This deformation amount was allowable $y$ axial deformation amount of the two-layer circular tube and was calculated by using the single circular tube model. The allowable $y$ axial deformation amount of acrylic plastic was $0.255 \mathrm{~mm}$ by substituting values on table 1 into eqn (16). Similarly, the allowable $y$ axial deformation amount of stainless steel was $0.188 \mathrm{~mm}$ by using eqn (17). In this case, therefore, allowable $y$ axial deformation amount of the two-layer circular tube became $0.188 \mathrm{~mm}$.

Von Mises stress distributions in the two-layer circular tube was simulated by FEA. The distributions of the stress show in fig. 8. We found that the maximum stress was occurred at a contact point with elastic body 2 in stainless steel. In the case of $y$ axial deformation amount $0.188 \mathrm{~mm}$, the maximum stress was $387 \mathrm{MPa}$ and was larger than the yield strength $\sigma_{a 1}=205 \mathrm{MPa}$ of stainless steel. We considered that the difference was caused by frictional influence. The allowable deformation as shown in eqn (16) and eqn (17) and the allowable load as shown in eqn (19) and eqn (20) are insecure sustainability in considering of frictional influence. 


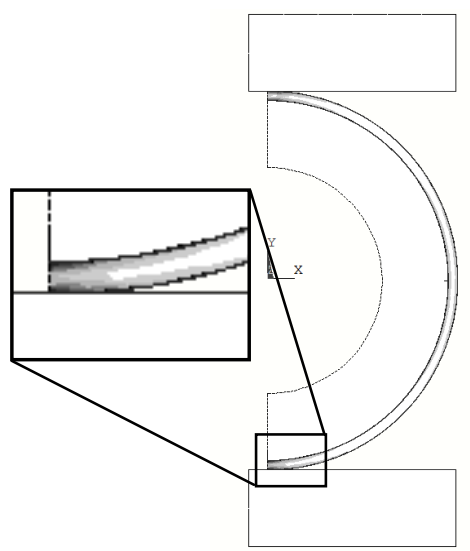

(a) In the case of $y$ axial deformation, the amount was $0.188 \mathrm{~mm}$

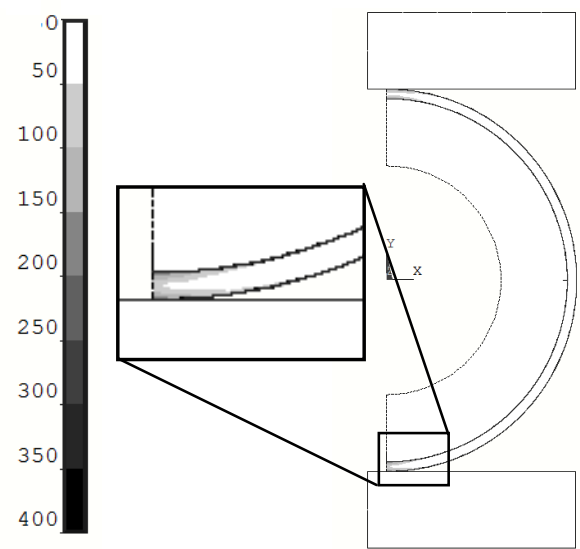

(b) In the case of $y$ axial deformation, the amount was $0.100 \mathrm{~mm}$.

Figure 8: $\quad$ Von Mises stress distribution in two-layer circular tube which was simulated by FEA (Unit: MPa).

On the other hand, in the case of $y$ axial deformation amount $0.100 \mathrm{~mm}$, the maximum stress was 201MPa and was smaller than the yield strength $\sigma_{a 1}=205 \mathrm{MPa}$ of stainless steel. Furthermore, $x$ axial deformation amounts were $0.086 \mathrm{~mm}$ inside of outer layer and $0.077 \mathrm{~mm}$ outside of inner layer. And the total reaction force was $435 \mathrm{~N}$.

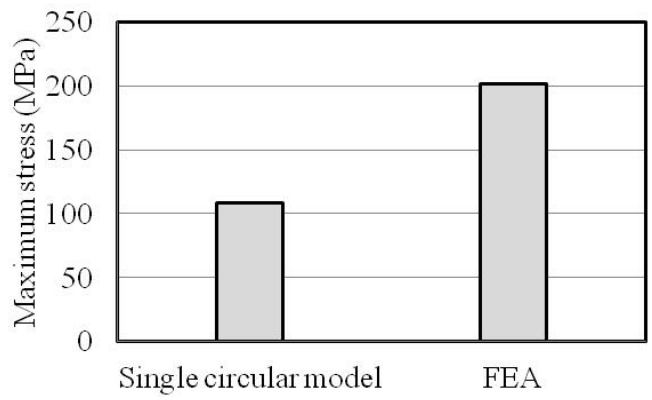

Figure 9: $\quad$ Comparison between the maximum von Mises stress simulated by FEA and the maximum bending stress by the single circular tube model in the case of y axial deformation amount $0.100 \mathrm{~mm}$.

By using the single circular tube model, the maximum bending stress was $109 \mathrm{MPa}$ by using eqn (6), the $x$ axial deformation amount was $0.092 \mathrm{~mm}$ by using eqn (8) and the total reaction force was $530 \mathrm{~N}$ by using eqns (19) and (20). Comparison between the results simulated by FEA and the results calculated by the single circular tube model were shown in figs 9-11. 


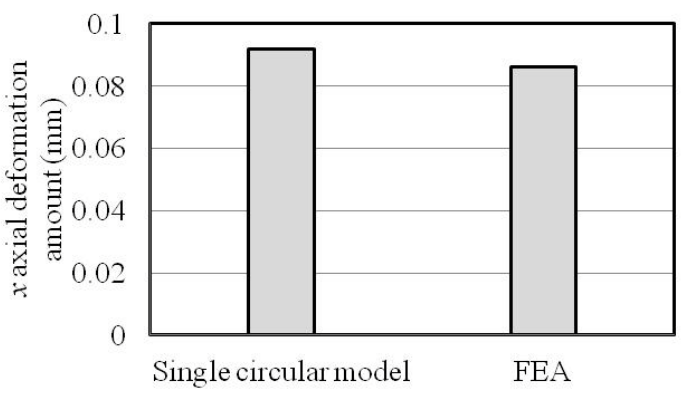

Figure 10: Comparison between the $\mathrm{x}$ deformation amount simulated by FEA and the $x$ deformation amount by the single circular tube model in the case of $y$ axial deformation amount $0.100 \mathrm{~mm}$.

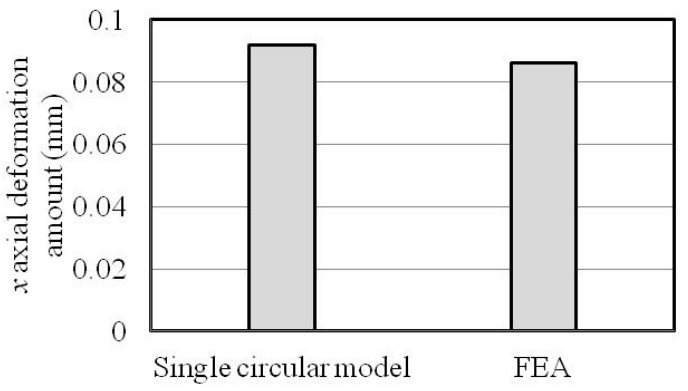

Figure 11: Comparison between the total reaction force simulated by FEA and total reaction force by the single circular tube model in the case of $y$ axial deformation amount $0.100 \mathrm{~mm}$.

The maximum von Mises stress simulated by FEA was larger than the maximum bending stress calculated by the single circular tube model as shown in fig. 9. The $x$ axial deformation amount of two-layer circular tube simulated by FEA was smaller than the $x$ axial deformation amount calculated by the single circular tube model as shown in fig. 10. One of the reasons is frictional influence. We consider that friction occurs between outer layer and inner layer near $y$ axis. It was thought that the $x$ axial deformation amount became small since the resistance i.e. total reaction force as shown in fig. 11 becomes large in consideration of frictional influence.

Therefore we need to use contact conditions of a two-layer circular tube by a single circular tube model (as shown in eqns (16), (17), (19) and (20)) with an appropriate correction in consideration of frictional influence.

\section{Conclusions}

We created a new seismic isolation device and tested the energy absorbing capacity of a two-layer circular tube which was a unit of the created device. And 
we could show contact conditions by using single circular tube model as design condition of sustainable two-layer circular tube. However we need to use the contact conditions with an appropriate correction in consideration of frictional influence. We were able to clarify that the relationships between the contact condition and external force by friction by using FEA.

\section{Acknowledgements}

This work was supported by Japan Science and Technology Agency promoting industry-academia collaborative R\&D based on the research output and IP generated by basic research "A-STEP (Adaptable and Seamless Technology Transfer Program through Target-driven R\&D) Feasibility Study (FS) Stage Exploratory Research”.

\section{References}

[1] Murota, N., Japan's Latest Earthquake-Resistance Technology, Highlighting Japan, April 2010, pp. 7-9, 2010.

[2] Taylor, A. and Aiken, I., What's Happened to Seismic Isolation of Buildings in the U.S.?, Structure magazine, March 2012, pp. 10-13, 2012.

[3] Ota, T. and Enoki, S, Material design of a biomimetic composite material used for a wooden building joint structure, WIT Transactions on Ecology and the Environment, 138, pp. 329-338, 2010.

[4] Enoki, S, Mechanical Property of Metal Lattice filled Low-Rigidity Material in the Cells [in Japanese], Proc. of 2012 Spring Annual Conference JSDE, pp. 23-24, 2012. 